\title{
ON THE EXISTENCE OF HIGH INDEX TOPOLOGICALLY MINIMAL SURFACES
}

\author{
DAVID BACHMAN ${ }^{1}$ AND Jesse JOHNSON ${ }^{2}$
}

\begin{abstract}
The topological index of a surface was previously introduced by the first author as the topological analogue of the index of an unstable minimal surface. Here we show that surfaces of arbitrarily high topological index exist.
\end{abstract}

Consider a compact, connected, two sided surface $S$ properly embedded in a compact, orientable 3-manifold $M$. The disk complex $\Gamma(S)$ is the simplicial complex defined as follows: Vertices of $\Gamma(S)$ are isotopy classes of compressing disks for $S$. A collection of $n$ such isotopy classes is an $(n-1)$-simplex of $\Gamma(S)$ if there are representatives of each that are pairwise disjoint.

1. Definition. If $\Gamma(S)$ is non-empty then the topological index of $S$ is the smallest $n$ such that $\pi_{n-1}(\Gamma(S))$ is non-trivial. If $\Gamma(S)$ is empty then $S$ will have topological index 0 . If $H$ has a well-defined topological index (i.e. $\Gamma(S)=\emptyset$ or some homotopy group of $\Gamma(S)$ is non-trivial) then we will say that $S$ is topologically minimal.

Topological index was introduced by the first author as the topological analogue of the index of an unstable minimal surface [Bacc], and later used in [Baca] and [Bacb] to prove various results about Heegaard splittings of 3-manifolds. Here we show this definition is not vacuous for high index surfaces by proving the following existence result:

2. Theorem. There is a closed 3-manifold, $M(1)$, with an index 1 Heegaard surface $S$, such that for each $n$, the lift of $S$ to some $n$-fold cover $M(n)$ of $M(1)$ has topological index $n$.

The manifold $M(1)$ of Theorem 2 is obtained by gluing together the boundary components of the complement of a link in $S^{3}$, which we will construct as follows:

We say $S^{2} \subset S^{3}$ is a bridge sphere for a knot or link $L \subset S^{3}$ if $L$ meets each of the balls bounded by $S^{2}$ in a collection of boundary parallel arcs. If the minimum number of such arcs is $b$, then we say $L$ is a $b$-bridge knot/link.

Throughout the paper, we will assume $L \subset S^{3}$ is a two component two-bridge link such that for a regular neighborhood $N$ of $L$ the complement $M=S^{3} \backslash N$ contains no essential planar surface with Euler characteristic greater than -3 .

By [HT85] or [BS05], such links can be constructed by choosing a sufficiently complicated braid to define the link. Let $S^{2}$ be a bridge sphere for $L$ that realizes its bridge number, and let $B^{ \pm}$be the balls in $S^{3}$ bounded by $S^{2}$. Let $S=\left(S^{2} \backslash N\right) \subset M$ and $C^{ \pm}=\left(B^{ \pm} \backslash N\right) \subset M$. The manifold $M(1)$ of Theorem 2 is obtained from $M$ by

\footnotetext{
Received by the editors September 14, 2009.

${ }^{1}$ Partially supported by NSF grant DMS-0906151.

${ }^{2}$ Partially supported by NSF MSPRF grant 0602368.
} 
gluing its boundary components together in such a way so that the surface $S$ glues up to a closed, orientable surface.

A boundary compressing disk for $S$ in $M$ is a disk $D$ with interior disjoint from $S$ and $\partial M$ such that $\partial D$ consists of an essential arc in $S$ and an arc in $\partial M$. Because the interior of $D$ is disjoint from $S$, the disk $D$ is contained in either $C^{-}$or $C^{+}$.

3. Lemma. Every compressing or boundary compressing disk for $S$ in $C^{-}$meets every compressing or boundary compressing disk for $S$ in $C^{+}$.

Proof. Assume for contradiction there are disjoint compressing or boundary compressing disks $E_{0}^{-} \subset C^{-}$and $E_{0}^{+} \subset C^{+}$for $S$. We will replace $E_{0}^{-}$and $E_{0}^{+}$with boundary compressing disks $E^{-}, E^{+}$as follows: The intersection $C^{-} \cap \partial M$ consists of two annuli. If $E_{0}^{-}$is a boundary compressing disk, then one of these two annuli contains an arc of $\partial E_{0}^{-}$. If this arc is essential in the annulus that contains it, then we will let $E^{-}=E_{0}^{-}$.

If this is not the case, or if $E_{0}^{-}$is a compressing disk, then $E_{0}^{-} \cap S$ is an arc with both endpoints in the same loop of $\partial S$ or a loop in $S$, respectively. In either case $E_{0}^{-} \cap S$ separates $S$ into two components, each of which is an annulus or a pair of pants. For each of these components, there is a boundary compressing disk for $S$ in $C^{-}$that intersects $\partial N \cap C^{-}$in an essential arc and intersects $S$ in this component. One of these components contains the $\operatorname{arc} E_{0}^{+}$. We will let $E^{-}$be the boundary compressing disk for the other component.

Thus we have chosen $E^{-}$to be disjoint from $E_{0}^{+}$and so that $E^{-} \cap \partial N$ is essential in $\partial N \cap C^{-}$. A similar construction produces a boundary compressing disk $E^{+}$in $C^{+}$ that is disjoint from $E^{-}$and intersects $\partial N \cap C^{+}$in an essential arc.

Recall that $M$ is the complement in $S^{3}$ of a regular neighborhood $N$ of $L$. We can extend each disk $E^{ \pm}$to a disk $D^{ \pm}$in $S^{3}$ whose boundary consists of an arc in $L$ and an arc in $S^{2}$. The disks $D^{+}, D^{-}$will either be disjoint or they will intersect in one or two points contained in $L \cap S^{2}$.

If the two disks intersect in one point in $L \cap S^{2}$ then we will slide the two arcs of $L$ across these disks into $S^{2}$. The resulting link $L^{\prime}$ is isotopic to $L$ and intersects $S^{2}$ in a point and an arc. We can isotope $L^{\prime}$ so that the arc of intersection becomes a single point. The resulting link has one bridge with respect to $S^{2}$, contradicting our assumption that $L$ is 2-bridge.

If the two disks intersect in two points in $L \cap S^{2}$ then we can again isotope the bridge $\operatorname{arcs}$ into $S^{2}$, so that the resulting link $L^{\prime}$ is isotopic to $L$ and consists of a one-bridge component and a component contained in $S^{2}$. Such a link is either a two component unlink or a Hopf link. In either case $M$ will contain an essential planar surface whose Euler characteristic is at least -2 .

Finally, if the two disks are disjoint then the frontier of a regular neighborhood of each $D^{ \pm}$in $B^{ \pm}$is a disk $A^{ \pm}$. The loops $\partial A^{+}$and $\partial A^{-}$are disjoint in the fourpunctured sphere $S^{2} \backslash L$, and each loop separates two of the punctures from the other two, so they must be parallel. If we isotope the disks so that their boundaries coincide, the resulting sphere separates $L$ into two one-bridge components, so $L$ is the two-component unlink. This is again a contradiction, as $M$ will contain an essential disk. This final contradiction implies that there are no disjoint disks $E_{0}^{-}, E_{0}^{+}$. 
Fix $n$. The intersection of the bridge surface $S$ with $\partial M$ defines a meridional slope in each component of $\partial M$. We will also choose an arbitrary longitude for each boundary component. For each $i \leq n$, let $M_{i}$ be a 3-manifold homeomorphic to $M$, and let $S_{i}$ and $C_{i}^{ \pm}$be the images of $S$ and $C^{ \pm}$in $M_{i}$. Define one component of $L$ to be the left component and call the other the right component. Each $M_{i}$ then has a left and right boundary component inherited from the left and right component, respectively, of $L$. The surface $S_{i}$ intersects each boundary component of $M_{i}$ in a pair of meridians, and each component of $\partial M_{i}$ inherits a special longitude from $M$.

Let $M(n)$ be the result of gluing the right component of each $\partial M_{i}$ to the left component of $\partial M_{i+1}$, as well as gluing the right component of $\partial M_{n}$ to the left component of $\partial M_{1}$. All gluings should be made so that meridians are sent to meridians and longitudes are sent to longitudes. Moreover, we will glue so that the surfaces $S_{i}$ together form a closed surface $S(n) \subset M(n)$, with $\bigcup C_{i}^{+}$a submanifold of $M$ whose boundary is $S(n)$. Because we use the same gluing along each pair of tori, the manifold $M(n)$ will be an $n$-fold cyclic cover of the manifold $M(1)$.

4. Lemma. For each $n$, the surface $S(n) \subset M(n)$ is a genus $n+1$ Heegaard surface.

Proof. The intersection of $L$ with $B^{+}$is a pair of unknotted arcs. Hence, there is a disk $D^{+} \subset B^{+}$with $\partial D^{+} \subset \partial B^{+}$such that $D^{+}$separates $B^{+}$into two components, each containing an unknotted arc of $L \cap B^{+}$. The disk $D^{+}$can be chosen disjoint from $N$ so that its image in each $M_{i}$ is a disk $D_{i}^{+} \subset C_{i}^{+}$. Because each component of $B^{+} \backslash D^{+}$contains an unknotted arc of $L \cap B^{+}$, the closure of $C_{i}^{+} \backslash D_{i}^{+}$is a pair of solid tori. We will call these the left and right solid tori of $\overline{C_{i}^{+} \backslash D_{i}^{+}}$, respectively as each meets the left and right boundary component of $M_{i}$.

When we glue $M_{i}$ to $M_{i+1}$, the right solid torus of $\overline{C_{i}^{+} \backslash D_{i}^{+}}$is glued to the left solid torus of $\overline{C_{i+1}^{+} \backslash D_{i+1}^{+}}$along an annulus that is primitive in each. Hence, these two together form a solid torus, which we denote $T_{i}$. Similarly, the right solid torus of $\overline{C_{n}^{+} \backslash D_{n}^{+}}$is glued to the left solid torus of $\overline{C_{1}^{+} \backslash D_{1}^{+}}$to form a solid torus $T_{n}$.

To reconstruct $\bigcup C_{i}^{+}$, for each $i<n$ we glue $T_{i}$ to $T_{i+1}$ along the disk $D_{i+1}^{+}$and we glue $T_{n}$ to $T_{1}$ along $D_{1}^{+}$. Hence, $\bigcup C_{i}^{+}$is a handlebody of genus $n+1$. A similar argument implies that $\bigcup C_{i}^{-}$is also a handlebody. The two handlebodies intersect along their common boundary $S(n)$, so $S(n)$ is a Heegaard surface for $M(n)$.

5. Proposition. The surface $S(n)$ has topological index at most $n$.

Proof. As in the proof of Lemma 4, the Heegaard surface $S(n)$ bounds handlebodies $\cup C_{i}^{+}$and $\bigcup C_{i}^{-}$, and there is a compressing disk $D_{i}^{+}$for $S(n)$ contained in each $C_{i}^{+}$ and a second compressing disk $D_{i}^{-}$with interior in $C_{i}^{-}$. For $i \neq j$, the subsets $C_{i}^{ \pm}$ and $C_{j}^{ \pm}$have disjoint interiors so $D_{i}^{ \pm}$and $D_{j}^{ \pm}$will be disjoint. If $i=j$ then the disks are either the same or they are on opposite sides of $S_{i}$, so as noted above they must intersect. In $\Gamma(S(n))$, there will thus be edges connecting the vertices corresponding to $D_{i}^{+}$and $D_{i}^{-}$to the vertices corresponding to $D_{j}^{+}$and $D_{j}^{-}$, if and only if $i \neq j$. Thus the subset $P$ of $\Gamma(S(n))$ spanned by these vertices is the $n$-fold suspension of the 0 -sphere, as in Figure 1.

We will define a map $F: \Gamma(S(n)) \rightarrow P$ as follows: Let $D$ be a compressing disk for $S(n)$ and assume, without loss of generality, that $D$ is contained in $\bigcup C_{i}^{+}$. Let $T$ be the image in $M$ of the torus boundary components of each $M_{i}$. Assume that we have 


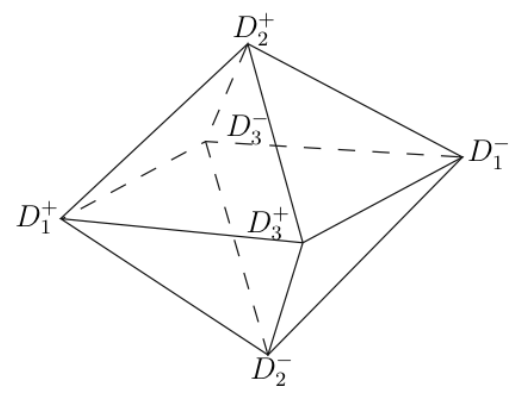

Figure 1. The complex $P$ when $n=3$.

isotoped $D$ so as to minimize $\partial D \cap T$. Because $T \cap S(n)$ and $\partial D$ are essential in $S(n)$, the result is canonical up to isotopy of $D \cup T$. (To see this, one can fix a hyperbolic metric on $S(n)$ and isotope $T \cap S(n)$ and $\partial D$ to their geodesic representatives in $S(n)$.) If $D$ is disjoint from $T$ then it is contained in the image of some $M_{i}$. In this case, $F$ will send $D$ to $D_{i}^{+}$.

Otherwise, note that $T$ is incompressible in $M$, so any innermost loop of $D \cap T$, bounding a disk in $D$, must also bound a disk in $T$. Thus we can isotope $D$ so that $D \cap T$ is a collection of arcs. Let $\alpha$ be an outermost arc that cuts off a disk $E \subset D$ whose interior is disjoint from $T$. Let $\beta \subset \partial D$ be the second arc making up $\partial E$. If $\beta$ is boundary parallel in $S(n) \backslash T$ then the isotopy of $\beta$ into $T$ determines an isotopy of $D$ that reduces $\partial D \cap T$. (The isotopy produces a loop of $D \cap T$ that can then be removed.) Thus we can assume that $\beta$ is essential in $S(n) \backslash T$. We will let $F$ send $D$ to $D_{i}^{+}$for the smallest $i$ such that there is such an outermost subdisk $E$ of $D \backslash T$ lying in $C_{i}^{+}$.

We will show that $F$ sends the endpoints of each 1-simplex in $\Gamma(S(n))$ to the endpoints of a 1-simplex in $P$. Assume $D$ and $D^{\prime}$ are disjoint compressing disks for $S(n)$ such that $F(D)$ is either $D_{i}^{+}$or $D_{i}^{-}$and $F\left(D^{\prime}\right)$ is either $D_{j}^{+}$or $D_{j}^{-}$. If $i \neq j$ then the disks $D_{i}^{ \pm}, D_{j}^{ \pm}$are disjoint and the edge from $D$ to $D^{\prime}$ can be sent to the edge from $D_{i}^{ \pm}$to $D_{j}^{ \pm}$, accordingly. We need to show that if $i=j$ then $D$ and $D^{\prime}$ must be on the same side of $S(n)$, and thus are both sent to the same vertex of $P$.

Assume for contradiction $D$ is on the positive side of $S(n)$ and $D^{\prime}$ is on the negative side. By definition, after an appropriate isotopy fixing their boundaries, $D \cap M_{i}$ will be a compressing or $\partial$-compressing disk $E$ for $S_{i}$ in $M_{i}$. Similarly, $D^{\prime} \cap M_{i}$ will be a compressing or $\partial$-compressing disk $E^{\prime}$. As $E$ and $E^{\prime}$ are on opposite sides of $S_{i}$, by Lemma 3 they must intersect. But $E$ and $E^{\prime}$ are subdisks of disjoint disks $D$ and $D^{\prime}$. This contradiction implies that $D$ and $D^{\prime}$ are on the same side of $S(n)$.

Note that whenever the complex $P$ contains the boundary of an $n$-simplex, it also contains its interior. It follows that since $F$ is defined on the 1-skeleton of $\Gamma(S(n))$, and the higher dimensional cells in $\Gamma(S(n))$ are determined by its 1-skeleton, the map $F$ can be extended over the rest of $\Gamma(S(n))$. 
The subcomplex $P$ defines an $(n-1)$-sphere in $\Gamma(S(n))$. The map $F: \Gamma(S(n)) \rightarrow P$ fixes $P$, and is thus a retraction of $\Gamma(S(n))$ onto an $(n-1)$-sphere. We conclude $\pi_{n-1}(\Gamma(S(n)))$ is non-trivial, and thus the topological index of $S(n)$ is at most $n$.

6. Lemma. If $F$ is a non-trivial, planar, topologically minimal surface in $M$ then $\chi(F) \leq-2$.

Proof. Let $F$ be a topologically minimal planar surface in $M$. If $F$ is essential then $\chi(F) \leq-3$ by our assumption on $M$. So we may suppose that $F$ is not essential, and is hence compressible. If all compressing disks are on the same side of $F$, then by [McC91], $\Gamma(F)$ is contractible, a contradiction. Furthermore, there must be vertices that represent compressions on opposite sides of $F$ that intersect, since otherwise $\Gamma(F)$ would be the join of two contractible complexes, and would thus be contractible. In particular, there must be a pair of essential loops in $F$ (the boundaries of these disks) that can not be isotoped to be disjoint. But any two essential loops in a planar surface with Euler characteristic at least -1 can be isotoped to be disjoint. Therefore $\chi(F) \leq-2$.

To complete the proof, we will need the following Theorem, which is proved in $[$ Bacc].

7. Theorem (Theorem 4.9 in [Bacc]). Let $F$ be a properly embedded, incompressible surface in an irreducible 3-manifold $M$. Let $S$ be a properly embedded surface in $M$ with topological index $n$. Then $S$ may be isotoped so that

(1) $S$ meets $F$ in $p$ saddles, for some $p \leq n$, and

(2) the sum of the topological indices of the components of $S \backslash N(F)$ in $M \backslash N(F)$, plus $p$, is at most $n$.

8. Proposition. The surface $S(n)$ has topological index at least $n$.

Proof. Assume for contradiction that $S(n)$ has topological index $k<n$. As in the proof of Proposition 5, let $T$ be the image of $\bigcup \partial M_{i}$ in $M(n)$. Let $U$ be a regular neighborhood of $T$. By Theorem 7, we can isotope $S(n)$ to a surface $S^{\prime}$ such that each component of $S^{\prime} \backslash U$ is a topologically minimal surface and the indices of these surfaces sum to at most $k$. We may also isotope $S^{\prime}$ further so that any boundaryparallel component of $S^{\prime} \backslash U$ is isotoped into $U$.

Suppose some loop of $S^{\prime} \cap \partial \bar{U}$ is trivial in $\partial \bar{U}$. An innermost such loop cannot bound a disk in $S^{\prime} \backslash U$, because such a disk would be boundary-parallel, and would have thus been isotoped into $U$. Hence, an innermost loop of $S^{\prime} \cap \partial \bar{U}$ that is trivial in $\partial \bar{U}$ must be parallel to a compressing disk for a component of $S^{\prime} \backslash U$. But such a compressing disk would be disjoint from all other compressing disks for $S^{\prime} \backslash U$, which implies that the disk complex for $S^{\prime} \backslash U$ is contractible. This contradicts the fact that $S^{\prime} \backslash U$ is topologically minimal. Thus $S^{\prime} \cap \partial \bar{U}$ must consist entirely of essential loops.

For each $i$, let $S_{i}^{\prime}=S^{\prime} \cap\left(M_{i} \backslash U\right)$. Since the Heegaard surface $S^{\prime}$ must meet every incompressible surface in $M(n), S_{i}^{\prime} \neq \emptyset$. As $S^{\prime}$ is separating, it must meet each component of $\partial \bar{U}$ in at least two loops. Thus, each surface $S_{i}^{\prime}$ has at least four boundary components.

We claim that for each $i, \chi\left(S_{i}^{\prime}\right)=-2$. Note that $S(n)$ was a union of $n$ fourpunctured spheres, each of which has Euler characteristic -2 . Thus, $\chi(S(n))$, and 
thus $\chi\left(S^{\prime}\right)$, is $-2 n$. If $\chi\left(S_{i}^{\prime}\right) \neq-2$ for some $i$, then for some $S_{j}, \chi\left(S_{j}\right) \geq-1$. But such a surface that has at least four boundary components must have at least one planar component. Since, by Theorem 7, each component of $S_{i}^{\prime}$ is topologically minimal, we thus violate Lemma 6.

By Theorem 7, the sum of the indices of the components of $S^{\prime} \backslash U$ is at most $k<n$. Thus, there is some $i$ such that the topological index of $S_{i}^{\prime}$ is zero. Because the boundary of $M$ consists of tori, every boundary compressible surface is either compressible or a boundary parallel annulus. As any boundary parallel component of $S_{i}^{\prime}$ would have been isotoped into $U$ we conclude every component of $S_{i}^{\prime}$ must be essential.

If $S_{i}^{\prime}$ is connected, then the fact that $\chi\left(S_{i}^{\prime}\right)=-2$ and $\left|\partial S_{i}^{\prime}\right| \geq 4$ implies $S_{i}^{\prime}$ is planar. This violates our assumption that $L$ was chosen so that it does not contain essential planar surfaces whose Euler characteristic is at least -2 . Furthermore, this assumption on $L$ and the fact that $\chi\left(S_{i}^{\prime}\right)=-2$ implies that if $S_{i}^{\prime}$ is not connected then it is the union of two punctured tori. But then we contradict the fact that $\left|\partial S_{i}^{\prime}\right| \geq 4$. We conclude $S(n)$ must have index at least $n$.

Propositions 5 and 8 together imply the topological index of $S(n)$ is precisely $n$. Since $n$ was an arbitrary integer, this proves Theorem 2.

\section{References}

[Baca] D. Bachman. Heegaard splittings of sufficiently complicated 3-manifolds I: Stabilization. Available at http://arxiv.org/abs/0903.1695.

[Bacb] D. Bachman. Heegaard splittings of sufficiently complicated 3-manifolds II: Amalgamation. Available at http://arxiv.org/abs/0904.0485.

[Bacc] D. Bachman. Topological Index Theory for surfaces in 3-manifolds. Geom. Topol., 14(1):585-609, 2010.

[BS05] D. Bachman and S. Schleimer. Distance and bridge position. Pacific J. Math., 219(2):221$235,2005$.

[HT85] A. Hatcher and W. Thurston. Incompressible surfaces in 2-bridge knot complements. Invent. Math., 79(2):225-246, 1985.

[McC91] D. McCullough. Virtually geometrically finite mapping class groups of 3-manifolds. J. Differential Geom., 33(1):1-65, 1991.

Mathematics Department, Pitzer College, 1050 N. Mills Ave, Claremont, CA 91711

E-mail address: bachman@pitzer.edu

Department of Mathematics, Oklahoma State University, Stillwater, OK 74078

E-mail address: jjohnson@math.okstate.edu 